\title{
A Resource Allocation Scheme to Achieve Fairness in TH-UWB Sensor Networks with Near-Far Effects
}

\author{
Ghasem Naddafzadeh Shirazi*, Peng-Yong Kong ${ }^{\dagger}$, Hwee-Xian Tan ${ }^{\ddagger}$, Ranjeet Kumar Patro*, \\ Mun-Choon Chan ${ }^{\ddagger}$ and Chen-Khong Tham ${ }^{\dagger *}$ \\ *Department of Electrical and Computer Engineering, National University of Singapore, Singapore. \\ ${ }^{\dagger}$ Institute for Infocomm Research, Agency for Science, Technology \& Research (A*STAR), Singapore. \\ ${ }^{\ddagger}$ School of Computing, National University of Singapore, Singapore.
}

\begin{abstract}
The inherent near-far effect in wireless networks causes nodes that are further away from the receiver to suffer from throughput degradation, as packets from nodes that are nearer are typically received with greater signal strengths. This unfair situation is traditionally overcome by power control. However, when power control is not feasible, for example in tiny sensor nodes with power-limited batteries, alternative solutions have to be utilized to achieve fairness in the network. In this paper, we propose $U$-LiBRA - an UWB Location Based Resource Allocation scheme to alleviate the contention between near and far nodes in a TH-UWB sensor network. $U$-LiBRA allocates different time slots to nodes that are at varying distances from the receiver, so that nodes that are further away from the receiver can achieve higher throughput than what they would typically obtain under the influence of the near-far effect. Simulation results show that $U$-LiBRA can effectively mitigate the near-far effect and improve fairness in the absence of power control.
\end{abstract}

\section{INTRODUCTION}

With advances in Ultra Wide Band (UWB) technology, it is now possible to achieve high data rate transmissions at low cost for short-to-medium range communications. As such, we can envisage the large-scale adoption of wireless sensor network applications in many aspects of our daily lives, such as home and health-care monitoring.

However, these low cost tiny sensors may not have sufficient computational power to realize sophisticated medium access control (MAC) protocols that have been proposed for UWB networks [1], [2]. Consequently, the simple Aloha MAC protocol [3] has been adopted for use in the IEEE 802.15.4a standard for UWB-based LR-WPANs (Low-Rate Wireless Personal Area Networks) [4]. In the contention-based slotted version of Aloha for Time-Hopping (TH) UWB sensor networks [5], collisions occur whenever more than one sensor transmits in the same time slot. When all the nodes use the same power for transmissions, nodes that are closer to the receiver typically have higher Signal-to-Interference-andNoise-Ratio (SINR) values than nodes that are further away. In the presence of capture, this leads to an unfair situation whereby transmitters that are located nearer to the receiver will always have higher throughput than transmitters that are placed further away.

The unfair situation caused by near-far effect in wireless networks is conventionally mitigated through the use of power control. However, tight power control is infeasible in tiny sensor nodes which are resource limited, and may also result

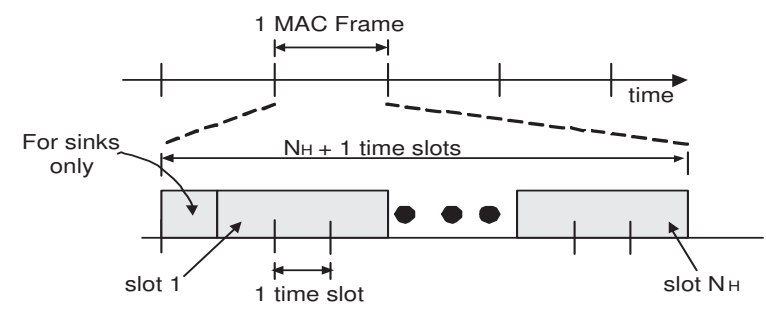

Fig. 1. Frame structure of Slotted-Aloha with TH-UWB

in lower network throughput [6]. In this paper, we propose $U$ LiBRA - an UWB Location Based Resource Allocation scheme to achieve fairness in a single-hop TH-UWB sensor network without power control. U-LiBRA alleviates the contention between near and far nodes by exploiting location information that is provided by the UWB physical layer technology to allocate segregated resources to these two groups of nodes. The efficacy of our proposed scheme is evaluated using various fairness metrics via simulations.

The rest of this paper is organized as follows. Section II discusses related work and motivation. The protocol description of $U-L i B R A$ is detailed in Section III. Evaluation results are presented in Section IV. This paper ends with concluding remarks in Section V.

\section{RELATED WORK AND MOTIVATION}

\section{A. Resource Allocation in a TH-UWB Sensor Network}

We consider a generic topology in which a set of $N$ transmitters (sensors) are distributed randomly around one receiver (sink). The nodes in the network make use of $p$-persistent Slotted-Aloha for medium access control. Note that we use the slotted version of Aloha because it usually outperforms the pure Aloha protocol [5], [7]. There are $N_{H}+1$ time slots in a MAC frame, as illustrated in Fig. 1. The first time slot is dedicated for transmissions originating from the sink, which is assumed to be reachable to the nodes within a single transmission hop. The remaining $N_{H}$ time slots are used by the sensors for transmissions to the sink. Each sensor node transmits with probability $p$ in an eligible slot.

We let $C=\left\{c_{1}, c_{2}, \ldots, c_{N_{H}}\right\}$ denote the set of time slots that are available for data transmissions to the sink in a TH-UWB sensor network. Each sensor $i$ is allocated a set 
of time slots $C_{i} \subseteq C$; if $c_{j} \in C_{i}$ then sensor $i$ can use slot $j$ for transmissions to the sink. In a network with $N$ sensor sources, the general allocation problem is to find the value of $\left\{C_{1}, C_{2}, \ldots, C_{N}\right\}$ such that fairness is maximized. Existing work in the literature suggests that finding such solution is NP-hard [8]-[10]. The high complexity of the optimal solution motivates the use of a simple heuristics to provide an acceptable suboptimal solution. In fact, the simplest method to overcome the original problem's complexity is to divide the nodes into two groups. We focus on this method throughout the paper and show that it provides acceptable fairness improvements, so dividing the nodes into more than two groups is unnecessary considering the complexity.

\section{B. Evaluating Fairness}

In general, a fairness metric is defined over a resource allocation set $X=\left\{x_{1}, x_{2}, \ldots, x_{N}\right\}$, where $0 \leq x_{i} \leq 1$ is sensor $i$ 's share from $X$ and $N$ is the total number of sensor nodes in the network. In this work, $X=\left\{P_{1}, P_{2}, \ldots, P_{N}\right\}$, where $P_{i}$ is the success probability of sensor $i$ in transmission to the sink. Note that since in the long-term throughput of each node is proportional to its success probability, $P_{i}$ represents the $i^{\text {th }}$ node's normalized throughput. In addition, $\sum_{x \in X} x$ can be used to indicate overall network throughput. Table I summarizes the fairness metrics that are used in this study. In this table, $\chi$ refers to the set of all possible resource allocation sets. A brief discussion on the fairness metrics is given in the next paragraph.

A resource allocation $X$ is called Max-min fair if it is impossible to increase any of the shares in $X$ without decreasing a smaller share in $X$ [11]. In the absence of extra constraints for the allocation, $X$ is Max-min fair if it has the largest minimum share (or allocation) among all possible allocations, $\chi$. Relative fairness [12] tries to minimize the variance of the fair allocation for any possible definition of fairness. Jain's fairness [13] considers the difference between the user shares. The maximum value of Jain's fairness metric is 1 , and it occurs when all the users have equal shares. As the variance of the values of the shares increases, Jain's fairness tends towards 0 . We define the two other fairness metrics (i.e. Group, Combined) for the situations that the nodes are divided into two groups, and we also use these metrics to measure the fairness improvement provided by our scheme. Specifically, Group fairness compares the difference between shares allocated to two groups of users, and tries to achieves fairness by balancing the group's allocation. Finally, Combined fairness provides a trade-off between fairness and achieving higher throughput. In other words, by increasing the value of $\alpha$ defined in Table I, the Combined fair allocation results in larger resource utilization (i.e. higher throughput).

For any fairness metric defined in Table I, the following two properties hold:

1) $0 \leq F(X) \leq 1 \quad \forall X \in \chi$

2) $X$ is fairer than $Y \Leftrightarrow F(X)>F(Y) \quad \forall X, Y \in \chi$

The fairness maximization problem is hence to find the best allocation $X^{*}$ such that one of the fairness metrics is maximized,

$$
X^{*}=\max _{X \in \chi} F(X) .
$$

Let $X_{0}$ be the random resource allocation set when all sensor nodes can uniform-randomly select any of the available time slots for transmission (i.e. $C_{i}=C, i=1,2, \cdots, N$ ). We define the fairness improvement achieved by $X^{*}$ over the allocation $X_{0}$ as

$$
I^{*}=I\left(F(.), X^{*}, X_{0}\right)=\frac{F\left(X^{*}\right)}{F\left(X_{0}\right)}
$$

Similarly, the throughput ratio of $X^{*}$ over $X_{0}$ is defined as

$$
A^{*}=\frac{\sum_{x \in X^{*}} x}{\sum_{x \in X_{0}} x}
$$

\section{U-LIBRA - PROTOCOL DESCRIPTION}

The optimal resource allocation scheme to achieve fairness in a TH-UWB sensor network is NP-hard and therefore impractical in realistic scenarios. In this section, we detail the protocol operations of $U-L i B R A$, a heuristic for resource allocation that can achieve fairness in polynomial time.

\section{A. Protocol Overview}

In $U$-LiBRA, all the $N$ sensors in the network are separated into two groups, $G_{1}$ and $G_{2}$, where $G_{1} \bigcap G_{2}=\emptyset$. The first group of $N_{1}$ sensors transmit with probability $\frac{p}{N_{H 1}}$ in the first $N_{H 1}$ slots (out of the available $N_{H}$ time slots). The remaining $N_{2}=N-N_{1}$ sensors transmit with with probability $\frac{p}{N_{H 2}}$ in the next $N_{H 2}=N_{H}-N_{H 1}$ time slots. The objective of $U$ LiBRA is hence to find the tuple $\left(N_{1}, N_{2}, N_{H 1}, N_{H 2}\right)$ that can maximize the overall network fairness. It should be noted that in contrast to the general allocation problem, the maximization problem in (1) is not NP-hard for $U$-LiBRA, and can be solved in polynomial time.

$U$-LiBRA estimates the throughput of a node by its probability of successful transmission, which is dependent on the distance between the transmitting node and the receiver. Using Theorem 1 in [14], the probability of a successful transmission of a sensor at distance $d_{0}$ from the receiver in a Rayleigh fading channel with Slotted-Aloha as the MAC protocol is given by:

$$
P_{s}=e^{-\frac{R_{t} N_{0} L\left(d_{0}\right)}{P_{0}}} \prod_{i=1}^{l} \frac{R_{t}\left(1-\frac{p}{N_{H}}\right)+\frac{L\left(d_{i}\right)}{L\left(d_{0}\right)}}{R_{t}+\frac{L\left(d_{i}\right)}{L\left(d_{0}\right)}}
$$

where $R_{t}$ is the SINR threshold value; $N_{0}$ is the noise power; $P_{0}$ is the transmit power; $L\left(d_{i}\right)$ is the pathloss of the $i^{t h}$ interferer at a distance $d_{i}$ from the receiver; and $l$ is the number of interferers.

$U$-LiBRA groups all the nodes in the network such that all the sensors in $G_{1}$ are closer to the sink than any of the sensors in $G_{2}$. Hence, the probability of successful transmission of an arbitrary sensor $j$ can be written as:

$$
P_{j}=e^{-\frac{R_{t} N_{0} L\left(d_{j}\right)}{P_{0}}} \prod_{i=k, i \neq j}^{N^{\prime}} \frac{R_{t}\left(1-\frac{p}{N_{H}^{\prime}}\right)+\frac{L\left(d_{i}\right)}{L\left(d_{j}\right)}}{R_{t}+\frac{L\left(d_{i}\right)}{L\left(d_{j}\right)}}
$$


TABLE I

FAIRNESS METRICS

\begin{tabular}{|c||c|}
\hline Name & Definition \\
\hline Max-min fairness [11] & $F(X)=\min (X)$ \\
\hline Relative fairness [12] & $F(X)=\min _{k=1,2, \ldots, N} \frac{Q_{k}(X)}{Q_{k}^{*}(X)}$ \\
& $\begin{array}{c}Q_{k}(X)=\sum_{i=1}^{k} x_{i} \text {, and } Q_{k}^{*}=\max _{X} \in \chi \\
\\
\end{array}$ \\
\hline should be sorted in ascending order.
\end{tabular}

where $k=1, N^{\prime}=N_{1}$ and $N_{H}^{\prime}=N_{H 1}$ if $j \in G 1$; and $k=N_{1}+1, N^{\prime}=N$ and $N_{H}^{\prime}=N_{H 2}$ if $j \in G 2$.

In the following subsections, we will detail the protocol operations of $U-L i B R A$, which include: (i) ranking algorithm; and (ii) tuple-search algorithm.

\section{B. Ranking Algorithm}

The sink collects the information about the nodes' distances to itself using a three-way handshake. During the network initialization, each sensor node $i$ transmits a LC (Location Control) message with a probability $p_{i}$ within a uniformrandomly selected time slot out of the $N_{H}$ available slots. The LC message contains the node ID, $i$, and the geographical location in the form $\left(x_{i}, y_{i}, z_{i}\right)$. Upon receiving each LC message, the sink calculates the distance of the node to itself. The sink then ranks the nodes in ascending order. After a waiting duration of $T_{L C W A I T}$, the sink broadcasts an AC (Allocation Control) message to the sensor sources. The AC message includes the IDs of all the nodes in the ranking list. An arbitrary node $i$ which has sent its LC message but whose ID is not in the AC message reduces its probability of transmission $p_{i}$ by multiplying it by some constant $0<\beta \leq 1$ to alleviate network collision, before retransmitting its LC message.

\section{Tuple-Search Algorithm}

The tuple-search algorithm is carried out at the sink if it does not receive a $\mathrm{LC}$ message for a duration of $T_{E A W A I T}$ after the ranking algorithm has been executed. The objective of the tuple-search algorithm is to find the optimal value of $\left(N_{1}, N_{2}, N_{H 1}, N_{H 2}\right)$ for an arbitrary topology, using a given fairness metric. The algorithm calculates the fairness obtained using each possible configuration of $\left(N_{1}, N_{2}, N_{H 1}, N_{H 2}\right)$ for $1 \leq N_{1} \leq N$ and then returns the configuration which provides the best fairness. Details of the tuple-search algorithm are highlighted in Algorithm 1.

After obtaining the values of the tuple $\left(N_{1}, N_{2}, N_{H 1}, N_{H 2}\right)$ with the best fairness, the sink transmits an EA (End Allocation) message containing the ranking and the optimal

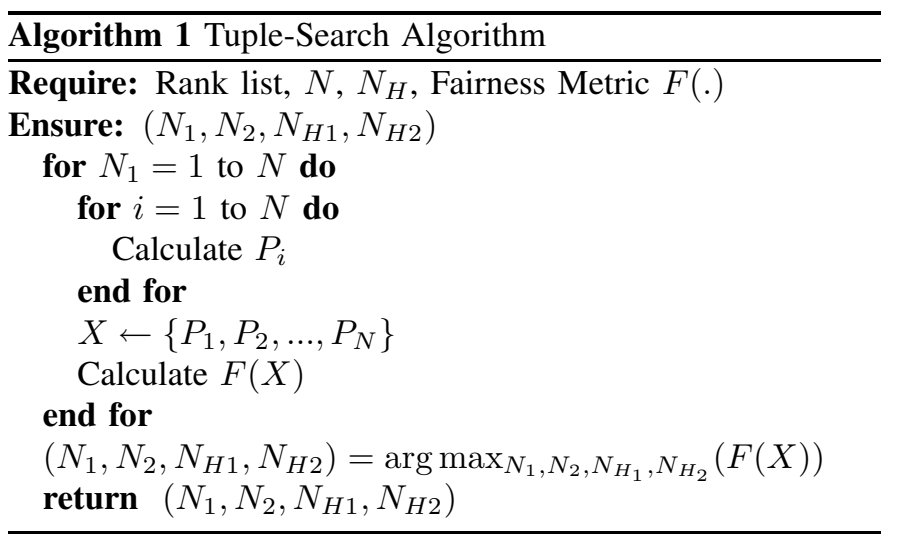

allocation information. The protocol terminates when all the sensors have received the EA message from the sink. The flowchart of $U-L i B R A$ is shown in Fig. 2.

\section{Performance Evaluation}

We use the Qualnet 4.0 simulator [15] to evaluate the efficacy of $U$-LiBRA over approximately 4000 randomly generated topologies. In each topology, the sink is located in the center of the terrain and the source nodes are randomly distributed around the sink. Table II details the values of the simulation parameters we have used.

The performance of $U$-LiBRA under the evaluation of different fairness metrics is illustrated in Table III. In this table, $S^{*}$ refers to the percentage of scenarios in which starvation occurs in at least one node ${ }^{1}$, i.e. $\exists x_{i}=0 ; I^{*}$ and $A^{*}$ refer to the average fairness improvement and throughput ratio, as defined in the equations (2) and (3) respectively; and $\mathcal{C}$ indicates the search complexity of Tuple-Search Algorithm using a particular fairness index.

It can be seen that the maximum average fairness improvement $I^{*}$ of 71.92 is achieved using the Group fairness index

\footnotetext{
${ }^{1}$ It should be noted that $9.67 \%$ of starvation in our simulation is unavoidable, because in this percentage of random topologies, number of nodes is much larger than number of available time slots, and starvation of some nodes always happens.
} 


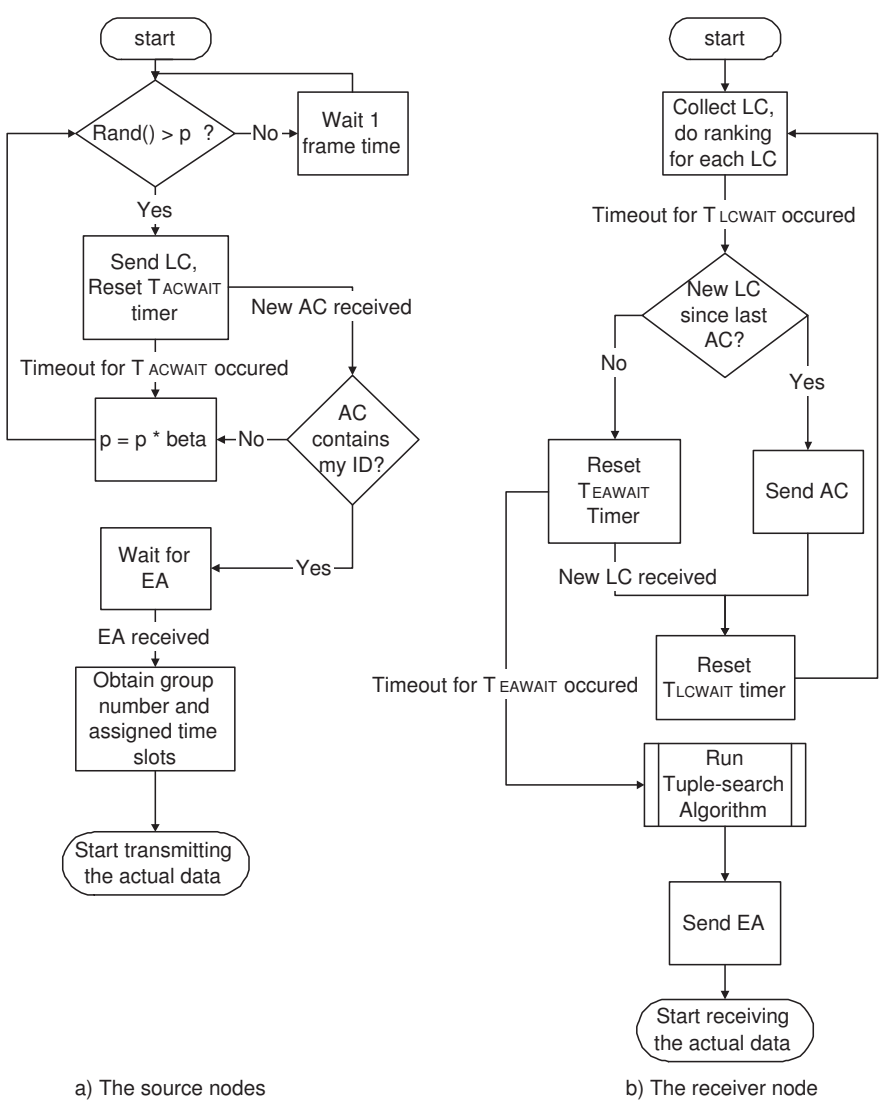

Fig. 2. Flowchart of $U-\operatorname{LiBRA}$ at a) the source nodes and b) the sink.

at a cost of starvation in $42.8 \%$ of the generated scenarios. This implies that in $42.8 \%$ of the topologies, the minimum difference between the success probabilities of $G_{1}$ and $G_{2}$ is achieved when some nodes have zero throughput. This starvation occurs because Group fairness favors minimum difference between groups and assigns relatively more time slots to nodes in $G_{2}$ (which are located further away from the sink), resulting in starvation in some of the nodes in $G_{1}$. This situation also happens when Jain's fairness is being used, in which a smaller difference in the success probabilities of the nodes results in better fairness.

Although Combined fairness takes into account the value of the total throughput to avoid starvation of nodes that are nearer to the sink, it tends to starve nodes that are further away, for large values of $\alpha$. Here, it should be noted that Combined fairness performs similarly to Group fairness for small values of $\alpha$. Therefore, choosing an appropriate value for $\alpha$ is critical to prevent starvation using the Combined fairness index. Max-min and Relative fairness metrics provide less fairness improvement (i.e. 1.12 and 1.03 respectively), but they have the advantage of avoiding starvation in the network whenever possible. Max-min fairness tries to improve the worst success probability while Relative fairness tries to improve the worst $k^{t h}$ prefix.

Taking into account all the results obtained, we select the Max-min fairness metric for further exploration, as it causes
TABLE II

SimUlation PARAMETERS

\begin{tabular}{|c||c|}
\hline \multicolumn{1}{|c||}{ Parameter } & Value \\
\hline Network Size $N$ & 1 to 128 \\
\hline Number of time slots, $N_{H}$ & $\{8,16,24,32\}$ \\
\hline Terrain Size & $8 \times 8 \mathrm{~m}^{2}$ \\
\hline Transmission Power $P_{0}$ & $-14.32 \mathrm{dBm}$ \\
\hline Noise Power $N_{0}$ & $-94 \mathrm{dBm}$ \\
\hline SINR threshold $R_{t}$ & $6 \mathrm{~dB}$ \\
\hline Channel Frequency $f$ & $4 \mathrm{GHz}$ \\
\hline Center Frequency $f_{c}$ & $4492.8 \mathrm{MHz}$ \\
\hline Pathloss model & $L(d)=d^{2}$ if $d \leq d_{0}, d_{0}=1 m$ \\
(IEEE 802.15.4a) & $L\left(d_{0}\right) . d^{1.79} \cdot\left(\frac{f}{f_{c}}\right)^{2}$ if $d>d_{0}$ \\
\hline Fading model & Rayleigh fading \\
\hline transmission probability $(p)$ & 0.9 \\
\hline$\beta$ & 0.9 \\
\hline Slot Length $\tau$ & $264 \mu s e c$ \\
\hline$T_{L C W A I T}$ & $3 \tau$ \\
\hline$T_{A C W A I T}$ & $16 \tau$ \\
\hline$T_{E A W A I T}$ & $24 \tau$ \\
\hline
\end{tabular}

TABLE III

FAIRNESS METRICS COMPARISON

\begin{tabular}{|c||c|c|c|c|}
\hline Fairness index & $S^{*}(\%)$ & $I^{*}$ & $A^{*}$ & $\mathcal{C}$ \\
\hline Max-min & 9.67 & 1.12 & 0.786 & $N_{H} \cdot N^{2}$ \\
\hline Relative & 9.67 & 1.03 & 0.947 & $N_{H}^{2} \cdot N^{4}$ \\
\hline Jain & 39.9 & 3.39 & 0.669 & $N_{H} \cdot N^{2}$ \\
\hline Group & 42.8 & 71.92 & 0.776 & $N_{H} \cdot N^{2}$ \\
\hline Combined & 75.5 & 13.18 & 1.135 & $N_{H} \cdot N^{2}$ \\
\hline
\end{tabular}

the least starvation, and it also has lower search complexity compared to Relative fairness. Fig. 3 shows the individual success probabilities of 16 source nodes with $N_{H}=8$ and using Max-min as the fairness metric. The last column indicated by "T" shows the average probability of success $T(X)=$ $N^{-1} \sum_{i=1}^{N} P_{i}$. In this example, the tuple $\left(N_{1}, N_{2}, N_{H_{1}}, N_{H_{2}}\right)$ that maximizes Max-min fairness is $(15,1,7,1)$, which means that $G_{1}$ has 15 nodes sharing a total of 7 time slots and $G_{2}$ has only 1 node using a single time slot. As compared to the original case (indicated by 'Before $U$-LiBRA') whereby all the sensors use all the 8 available time slots for transmissions, $U$ LiBRA alleviates network starvation and improves fairness by $77 \%$ without significant throughput deterioration.

Fig. 4 shows the average fairness improvement and throughput ratio as a function of network size. The maximum average improvement in fairness is around 2 when the number of nodes is 32 . In addition, the throughput achieved by $U-L i B R A$ is at least $50 \%$ of that without resource allocation. The above results suggest that dividing into two groups can efficiently improve fairness and hence the division of nodes into more than two groups incurs unnecessary complexity.

The running time of $U-L i B R A$ is illustrated in Fig. 5. As the network size $N$ increases, the protocol time increases linearly, 


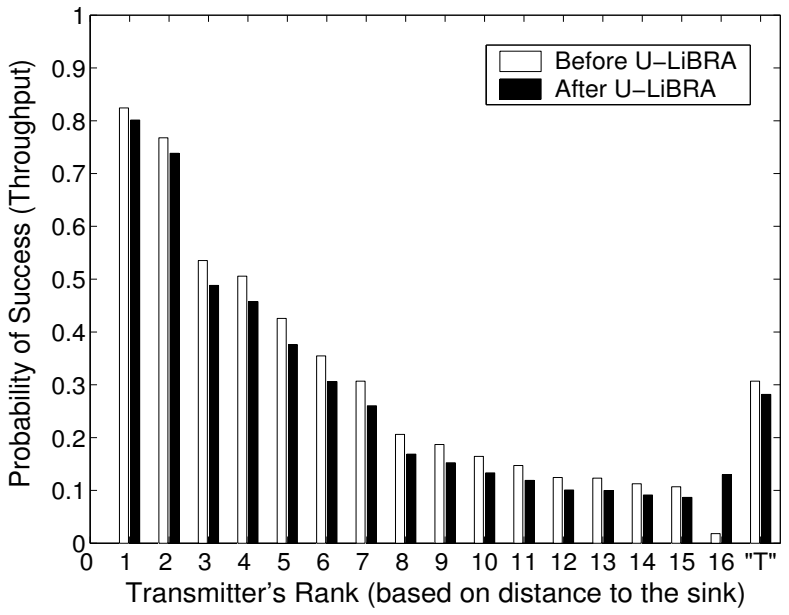

Fig. 3. Probability of successful transmission before and after resource allocation. The last column indicated by " $\mathrm{T}$ " shows the average probability of success, $T(X)$. The corresponding tuple for resource allocation is $(15,7,1,1) . I^{*}=1.77$ and $A^{*}=0.92$.

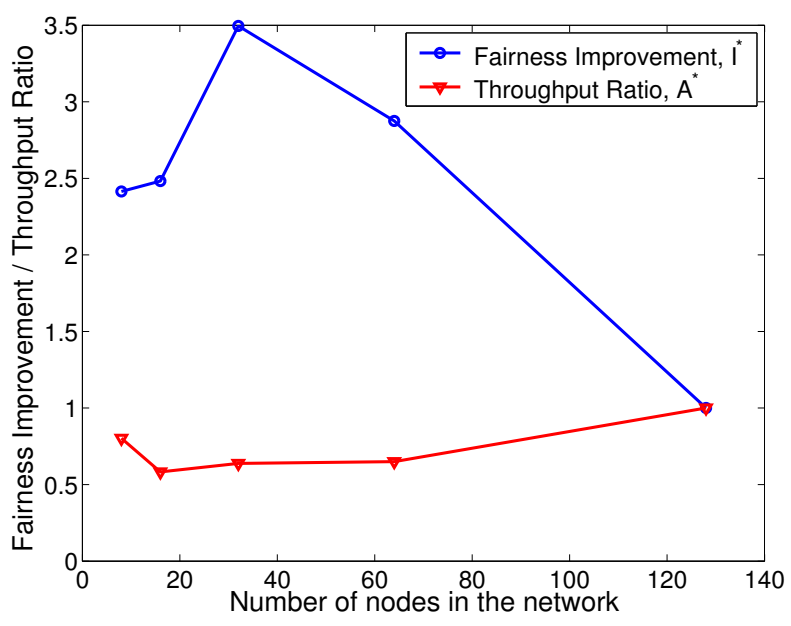

Fig. 4. Fairness Improvement and Throughput Ratio Vs. Network Size

thus proving the scalability of our proposed protocol.

\section{CONClusion}

This paper studies fairness in the absence of power control in a TH-UWB sensor network using Slotted-Aloha as the MAC protocol. By allocating segregated resources (time slots) to nodes which are located at different distances from the sink, $U$-LiBRA alleviates contention between nodes that are near to the sink from those that are further away. The effectiveness of our approach is studied using various fairness metrics through simulations, which result in different optimum resource allocations. In addition, Max-min fairness has been shown to provide the best tradeoff between fairness and throughput.

\section{ACKNOWLEDGMENT}

This work is done under the USCAM-CQ project which is a part of the Ultra Wide Band Sentient Computing Research Programme funded by Science and Engineering Research

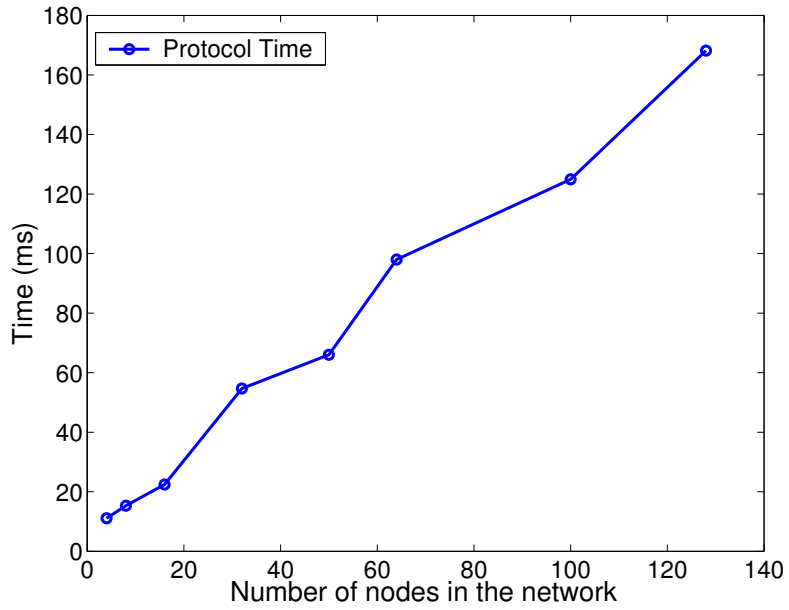

Fig. 5. Protocol running time versus number of source nodes.

Council (SERC), Agency for Science, Technology and Research, (A*STAR), Singapore.

\section{REFERENCES}

[1] P. Y. Kong and M. R. Shajan, "A Medium Access Control Protocol for Ultra-Wideband Wireless Ad Hoc Networks," in IEEE Vehicular Technology Conference (VTC), vol. 3, 2005, pp. 1993 - 1997.

[2] J. Zhu and A. O. Fapojuwo, "A complementary code-CDMA-based MAC protocol for UWB WPAN system," Eurasip Journal on Wireless Communications and Networking, vol. 2005, no. 2, 2005.

[3] N. Abramson, "The ALOHA System - Another Alternative for Computer Communications," in AFIPS Fall Joint Computer Conference, 1970, pp. 281-285.

[4] Wireless Medium Access Control (MAC) and Physical Layer (PHY) Specification for Low-Rate Wireless Personal Area Networks (LRWPANs), IEEE Std. Draft 802.15.4a, 2006.

[5] H. X. Tan, R. K. Patro, M. C. Chan, P. Y. Kong, and C. K. Tham, "Performance of Slotted-Aloha over TH-UWB," in IEEE International Conference on Ultra-WideBand (ICUWB 2007), 2007, pp. 714 - 719.

[6] J. Jeong, D. Culler, and J. H. Oh, "Empirical Analysis of Transmission Power Control Algorithms for Wireless Sensor Networks," in International Conference on Networked Sensing Systems (INSS), 2007, pp. 27 -34 .

[7] M. D. Benedetto, L. D. Nardis, G. Giancola, and D. Domenicali, "The aloha access (UWB) ${ }^{2}$ protocol revisited for IEEE 802.15.4a," ST Journal of Research, vol. 4, no. 1, pp. 131-142, 2007.

[8] A. M. Chou and V. O. K. Li, "Fair Spatial TDMA Channel Access Protocols for Multihop Radio Networks," in Annual Joint Conference of the IEEE Computer and Communications Societies (INFOCOM), 1991, pp. $1064-1073$

[9] D. Chakrabarty, A. Mehta, and V. Nagarajan, "Fairness and Optimality in Congestion Games," in ACM Conference on Electronic Commerce, 2005, pp. $52-57$.

[10] H. X. Tan, M. C. Chan, P. Y. Kong, and C. K. Tham, "A Resource Allocation Scheme for TH-UWB Networks with Multiple Sinks," in IEEE Wireless Communications and Networking Conference (WCNC), 2008, pp. $1547-1552$.

[11] D. Bertsekas and R. Gallager, "Max-min flow control," in Data Networks. Prentice Hall, 1987, ch. 6.5.2.

[12] A. Goel, A. Meyerson, and T. A. Weber, "Fair Welfare Maximization," in Social Science Research Network (SSRN) eLibrary, 2004.

[13] R. K. Jain, D. M. W. Chiu, and W. R. Hawe, "A Quantitative Measure of Fairness and Discrimination for Resource Allocation in Shared Computer Systems," DEC, Research Report TR-301, 1984.

[14] X. Liu and M. Haenggi, "Throughput Analysis of Fading Sensor Networks with Regular and Random Topologies," Eurasip Journal on Wireless Communications and Networking, vol. 5, no. 4, 2005.

[15] Qualnet Network Simulator, Scalable Network Technologies, Inc. [Online]. Available: www.qualnet.com 